\title{
Aplicación de técnicas de fitorremediación en suelos contaminados con plomo y cadmio
}

Application of phytoremediation techniques in soils contaminated with lead and cadmium

1 María Soledad Núñez Moreno Escuela Superior Politécnica de Chimborazo (ESPOCH), Facultad de Ciencias. Riobamba, Ecuador.

Solenu0408@gmail.com

Artículo de Investigación Científica y Tecnológica Enviado: 10/12/2021

Revisado: $15 / 01 / 2022$

Aceptado: 04/02/2022

Publicado:07/03/2022

DOI: https://doi.org/10.33262/concienciadigital.v5i1.3.2089

\section{contaminados con plomo y cadmio . ConcienciaDigital, 5(1.3), 6-25.}

Cítese: $\quad$ https://doi.org/10.33262/concienciadigital.v5i1.3.2089

CONCIENCIA DIGITAL, es una Revista Multidisciplinar, Trimestral, que se publicará en soporte electrónico tiene como misión contribuir a la formación de profesionales competentes con visión humanística y crítica que sean capaces de exponer sus resultados investigativos y científicos en la misma medida que se promueva mediante su intervención cambios positivos en la sociedad. https://concienciadigital.org

La revista es editada por la Editorial Ciencia Digital (Editorial de prestigio registrada en la Cámara Ecuatoriana de Libro con No de Afiliación 663) www.celibro.org.ec 
Palabras claves:

fitorremediación de suelos, técnica de fitoestabilización, fitorremediación con Tradescantia pallida,

fitorremediacion de $\mathrm{Pb}$ y $\mathrm{Cd}$.
Keywords: soil phytoremediation, phytostabilization technique, Tradescantia pallida

\section{Resumen}

El artículo tiene por objetivo analizar aplicaciones de técnicas de fitorremediación en suelos contaminados con plomo $(\mathrm{Pb})$ y cadmio (Cd), con el fin de seleccionar una potencial especie vegetal que sembrada en suelos degradados permita la absorción de los metales mencionados. Para esto, se aplicó a los procesos de fitorremediación conceptos como el factor de bioconcentración (FBC) y factor de translocación (FT). En el desarrollo de la investigación fueron presentadas las características del suelo, tipo de composta o enmienda orgánica, metodología y evaluación de datos; de los procesos de fitorremediación mediante las especies vegetales Lolium perenne, Poa pratensis, Maíz, Pennisetum setaceum y Tradescantia pallida; sembradas en diferentes tipos de suelo. Evaluados los datos se procedió al análisis con las especies Lolium perenne, Maíz y Tradescantia pallida, ya que presentaron las mejores propiedades. Como resultado de la aplicación de las técnicas de fitorremediación en las tres especies, se obtiene que la planta Tradescantia pallida al tener valores de concentración total de $\mathrm{Pb}$ de 330,9 mg/kg y Cd 21,23 mg/kg, FBCraíz de 0,92 y FT de 0,53 para Pb, FBCraíz de 22,1 y FT de 0,2 para $\mathrm{Cd}$; alcanza las mejores propiedades para retener $\mathrm{Pb}$ y $\mathrm{Cd}$ por la técnica de fitoestabilización, en comparación con las plantas de Maíz y la Tradescantia pallida. La especie vegetal Tradescantia pallida, a pesar de que fue sembrada en un suelo antropogénico con elevado contenido de contaminación, ya que fue un antiguo botadero de basura, logró acumular valores de concentración de $\mathrm{Pb}$ en la parte aérea de $114,1 \mathrm{mg} / \mathrm{kg}$ y de $\mathrm{Cd}$ en la raíz de $17,03 \mathrm{mg} / \mathrm{kg}$ que, en comparación con las otras especies vegetales investigadas, tienen una mejor concentración de metales, razón por la cual, puede seleccionarse para la remediación de suelos contaminado con $\mathrm{Pb}$ y $\mathrm{Cd}$.

\section{Abstract}

The article aims to analyze applications of phytoremediation techniques in soils contaminated with lead $(\mathrm{Pb})$ and cadmium $(\mathrm{Cd})$, to select a potential vegetal specie that, planted in degraded soils, allows the absorption of the mentioned metals. For this, concepts such as bioconcentration factor (BCF) and 
phytoremediation, $\mathrm{Pb}$ and $\mathrm{Cd}$ phytoremediation. translocation factor (TF) were applied to phytoremediation processes. In the development of the research, the characteristics of the soil, type of compost or organic amendment, methodology and data evaluation were presented; of the phytoremediation processes through the vegetal species Lolium perenne, Poa pratensis, Corn, Pennisetum Setaceum and Tradescantia Pallida; planted in different types of soil. Once the data was evaluated, it was analyzed with the species Lolium Perenne, Corn and Tradescantia Pallida, since they presented the best properties. As a result of the application of phytoremediation techniques in the three species, it is obtained that the Tradescantia pallida plant has values of total concentration of $\mathrm{Pb}$ of $330,9 \mathrm{mg} / \mathrm{kg}$ and $\mathrm{Cd} 21,23 \mathrm{mg} / \mathrm{kg}$, FBCroot of 0,92 and FT of 0,53 for $\mathrm{Pb}$, FBCroot of 22,1 and FT of 0,2 for $\mathrm{Cd}$; achieved the best properties to retain $\mathrm{Pb}$ and $\mathrm{Cd}$ by the phytostabilization technique, compared to corn plants and Tradescantia pallida. The vegetal specie Tradescantia pallida, although it was sown in an anthropogenic soil with a high content of contamination, since it was an old open-air garbage dump, it accumulated values of $\mathrm{Pb}$ concentration in the aerial part of $114,1 \mathrm{mg} / \mathrm{kg}$ and $\mathrm{Cd}$ in the root of $17,03 \mathrm{mg} / \mathrm{kg}$, which in comparison with the other vegetal species investigated, have a better concentration of metals, therefore, it can be selected for the remediation of soils contaminated with $\mathrm{Pb}$ and $\mathrm{Cd}$.

\section{Introducción}

El crecimiento vertiginoso de la población mundial desde el año de 1950 con 2800 millones de personas hasta la actualidad con 7800 millones, es una problemática del presente y futuro del planeta, ya que, esto trae consigo una acelerada extracción de materias primas como metales, petróleo, gas, entre otros, que son procesadas por las industrias para la fabricación de productos dejando entre la extracción y la manufactura, contaminación de suelos, ríos, manantiales, acuíferos, mares y en general el medioambiente. Siendo, como lo menciona La Organización de las Naciones Unidas para la Alimentación y la Agricultura, la extracción de metales por parte de la industria minera una de las principales fuentes de contaminación del suelo de manera puntual y difusa, lo que representa un riesgo para la salud humana. 
Los metales son obtenidos a partir del procesamiento de roca mineral, realizada por la industria minera, junto con ello, se generan desperdicios como lixiviados, material particulado, jales de mina, escoria, entre otros, causantes de la contaminación y degradación de suelos. Si el crecimiento poblacional sigue en aumento la demanda de metales también crecerá, por lo tanto, se podría esperar que los suelos sigan contaminándose si no se integran estrictos controles técnicos a la minería para evitar su degradación (Rodríguez-Eugenio et al., 2019). En la figura 1 se muestra el crecimiento de la producción de metales pesados como Al, $\mathrm{Pb}, \mathrm{Cu}, \mathrm{Zn}$ y Cd, desde el año de 1998 hasta 2019, si estos metales, junto con otros, siguen extrayéndose aceleradamente el impacto a los suelos también seguirá en aumento.

Figura 1

Producción de metales por toneladas

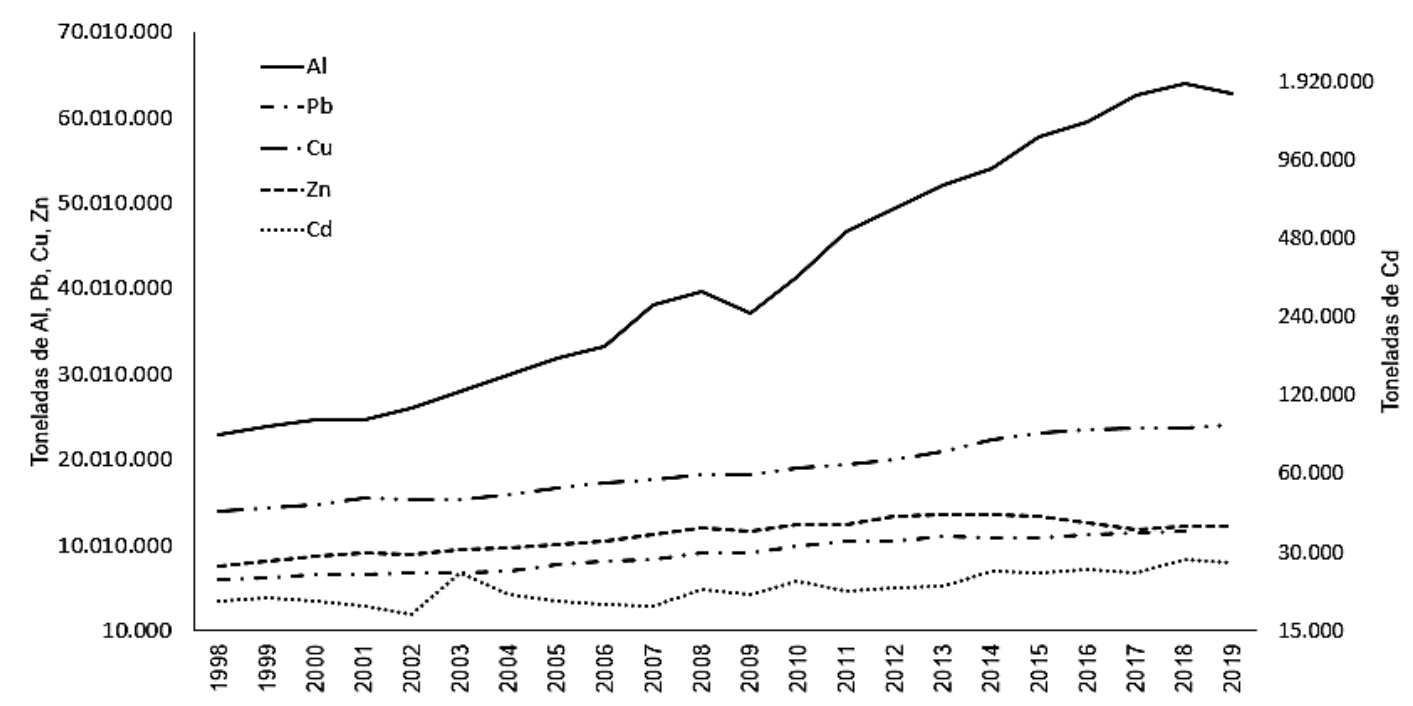

Fuente: Elaboración propia basada en MineralsUK (2022)

Ante esta problemática se propone una estrategia como la fitorremediación que utiliza conceptos de acumulación, extracción, degradación o volatilización, para absorber metales pesados, metaloides, polímeros, elementos y compuestos de suelo contaminados, mediante la siembra de una especie vegetal. La estrategia ganó aceptación en el año de 1990 al tratarse de una eficiente y económica forma de descontaminar suelos mediante diferentes tipos de plantas (Ashraf et al., 2010; Jaramillo, 2021).

La fitorremediación es una alternativa a las técnicas clásicas de descontaminación de suelos, se aplica para remediar contaminación generada por metales, aguas residuales, hidrocarburos, plaguicidas y toda clase de contaminación antropomórfica, teniendo la ventaja de ser potencialmente económica, ya que permite tratar al suelo sin necesidad de excavarlo y dirigirlo a otro lugar para su descontaminación, además de, solo requerir la 
siembra y cuidado de una especie vegetal, entre sus desventajas se considera tiempos prolongados para el crecimiento de la especie (Ávila, 2017; Volke \& Velasco, 2002).

En el presente trabajo se comparó las especies vegetales Lolium perenne, Poa pratensis, Maíz, Pennisetum setaceum y Tradescantia pallida, con el propósito de seleccionar la especie que alcanza la mejor retención $\mathrm{Pb}$ y $\mathrm{Cd}$ en sus órganos.

En el desarrollo de este tema de investigación se utilizó una metodología descriptiva, exploratoria y analítica, donde se estructuro un proceso de revisión, comparación y análisis de fuentes de investigación presentadas por: Amezcua-Ávila et al. (2020), fitorremediación de residuos de minas contaminados con metales pesados. Munive et al. (2018), fitorremediación con Maíz (Zea mays L.) y compost de Stevia en suelos degradados por contaminación con metales pesados. Pajoy (2017), potencial fitorremediador de dos especies ornamentales como alternativa de tratamiento de suelos contaminados con metales pesados, entre otras.

\section{Factor de bioconcentración y factor de translocación}

La capacidad que tiene las especies vegetales para retener metales en su raíz o acumular metales en su parte aérea (tallos + hojas o solo hojas), son evaluadas mediante el factor de bioconcentración (FBC) y el factor de translocación (FT), respectivamente. A partir de estos factores se realizará el análisis de resultados a las especies vegetales investigadas (Lokeshwari \& Chandrappa, 2006).

La tabla 1 presenta parámetros como FBC y FT que son evaluados para determinar si una especie vegetal utiliza técnicas de fitoextracción y/o fitoestabilización, en el proceso de fitorremediacion de suelos contaminados con metales pesados. También se presenta las características que tiene cada técnica de fitorremediación.

Tabla 1

Parámetros para la evaluación de las técnicas de fitoextracción y/o fitoestabilización

\begin{tabular}{|c|c|c|}
\hline \multirow[b]{2}{*}{ Parámetros } & \multicolumn{2}{|c|}{ Técnicas de Fitorremediación } \\
\hline & Fitoextracción & Fitoestabilización \\
\hline FT = Caérea / Craíz & FT $>1$ & FT $<1$ \\
\hline FBCraíz = Craíz / Csuelo & & \\
\hline $\begin{array}{l}\text { FBCaérea = Caérea } / \\
\text { Csuelo }\end{array}$ & FBCraíz $<1$ o FBCaérea $>1$ & FBCraíz $>1$ o FBCaérea $<1$ \\
\hline
\end{tabular}

\section{Csuelo}

Características

Elevada translocación de metales desde la raíz Nula o baja la translocación de metales desde hacia sus hojas y tallos, por lo que la la raíz hacia sus hojas y tallos, por lo que la acumulación es eficiente. Mientras que en la acumulación es deficiente. Mientras que en la raíz no se acumula metales o si lo hace es en raíz inmoviliza o secuestra el metal. La planta bajas cantidades. La planta es acumuladora. es exclusora.

FT = Factor de Translocación, Caérea = Concentración de metal en tallos + hojas o solo en hojas, Craíz = Concentración de metal

Csuelo = Concentración de metales en el suelo, FBCraíz = Factor de bioconcentración en la raíz

Csuelo = Concentración de metales en el suelo, FBCraíz = Factor de biocon
FBCaérea $=$ Factor de bioconcentración en tallos + hojas o solo en hojas

Fuente: Elaboración propia basada en: Jaramillo (2021), Martínez (2020), Medina \& Montano (2014) \& Wang et al. (2019) 
Fitorremediación en suelos contaminados con plomo y cadmio mediante especies vegetales

En este capítulo se presentarán las características del suelo, tipo de composta o enmienda orgánica, metodología y evaluación de los datos obtenidos, de los procesos de fitorremediación mediante las especies vegetales Lolium perenne, Poa pratensis, Maíz, Pennisetum setaceum y Tradescantia pallida, sembradas en diferentes tipos de suelo, para posteriormente realizar el respectivo análisis de resultados mediante el FBC y FT.

Fitorremediación mediante lolium perenne y poa pratensis en el jal de mina Dos Carlos

La fitorremediación con las especies vegetales Lolium perenne y Poa pratensis, en el jal de mina Dos Carlos, tuvo varias experimentaciones, de las cuales, para este artículo, se seleccionaron los experimentos de las dos especies más la adición de composta, debido a su elevada concentración de $\mathrm{Pb}$ y $\mathrm{Cd}$ en comparación con los demás experimentos. La fitorremediación pretende reducir los residuos de metales en el suelo del jal de mina Dos Carlos, generados por la minera local, y que se evidenciaron en las características de su suelo, el mismo que fue agregado composta para mejorar la absorción de metales. Para la siembra y cosecha se siguió una metodología que permitió evaluar la concentración de $\mathrm{Pb}$ y $\mathrm{Cd}$.

\section{Características del suelo del jal de mina Dos Carlos}

La extracción de metales a partir de la trituración del mineral de roca de la mina Dos Carlos generó un jal de mina, constituido con residuos de rocas, agua y reactivos químicos. Estos residuos ocasionaron que en la superficie del jal florezca poca o nada vegetación, además de, partículas de metales pesados, causantes de daños ambientales en los alrededores de la localidad. Por tal motivo, en el jal de mina se aplicó un proceso de fitorremediación para extraer la mayor cantidad de metales en el suelo contaminado (Amezcua-Ávila et al., 2020).

El jal de mina Dos Carlos está ubicado al sureste de la ciudad de Pachuca, México, tiene una extensión de 23 hectáreas y $20 \mathrm{~m}$ de altura, su suelo es semiárido con un clima templado y lluvias en verano. Las características del suelo del jal se presentan en la Tabla 2, las cuales fueron obtenidas a partir de muestras recolectadas a $10 \mathrm{~cm}$ del suelo (Amezcua-Ávila et al., 2020). 
Tabla 2

Características del suelo del jal de mina Dos Carlos

\begin{tabular}{lc}
\hline Características & Valores \\
\hline Textura del suelo & Franco arenoso \\
pH & 3,4 a 7,9 \\
$\mathbf{M O} \%$ & 0,27 \\
$\mathbf{N}$ inorgánico $(\mathrm{mg} / \mathrm{kg})$ & 5,6 a 24,1 \\
$\mathbf{P}(\mathrm{mg} / \mathrm{kg})$ & 1,2 a 18 \\
$\mathbf{K}(\mathrm{mg} / \mathrm{kg})$ & 32 a 252 \\
$\mathbf{C a}(\mathrm{mg} / \mathrm{kg})$ & 3672 a 10521 \\
$\mathbf{M g}(\mathrm{mg} / \mathrm{kg})$ & 64 a 679 \\
$\mathbf{C d}(\mathrm{mg} / \mathrm{kg})$ & 0,73 \\
$\mathbf{P b}(\mathrm{mg} / \mathrm{kg})$ & 14,34 \\
$\mathbf{Z n}(\mathrm{mg} / \mathrm{kg})$ & 57,88 \\
$\mathbf{N i}(\mathrm{mg} / \mathrm{kg})$ & 1,05 \\
$\mathbf{C u}(\mathrm{mg} / \mathrm{kg})$ & 6,51 \\
$\mathbf{M n}(\mathrm{mg} / \mathrm{kg})$ & 68,87 \\
\hline
\end{tabular}

Fuente: Amezcua et al. (2020)

Composta agregada al jal de mina Dos Carlos

La adición de composta agregada al suelo del jal de mina, para mejorar el crecimiento y la absorción de metales en las especies vegetales Lolium perenne y Poa pratensis, se la realizó a partir de alfalfa y excreta de borrego (Amezcua-Ávila et al., 2020). En la tabla 3 se presentan las características de la composta agregada al suelo del jal de mina Dos Carlos.

Tabla 3

Características de la composta agregada al suelo del jal de mina

\begin{tabular}{cccccccccccc}
\hline $\mathbf{p H}$ & $\begin{array}{c}\text { Materia } \\
\text { orgánica } \\
\%\end{array}$ & $\begin{array}{c}\mathbf{C} \\
\%\end{array}$ & $\begin{array}{c}\mathbf{N} \\
\%\end{array}$ & $\begin{array}{c}\mathbf{P} \\
(\mathrm{mg} / \mathrm{kg})\end{array}$ & $\begin{array}{c}\mathbf{C O 2} \\
(\mathrm{mg} / \mathrm{g})\end{array}$ & $\begin{array}{c}\mathbf{C d} \\
(\mathrm{mg} / \mathrm{kg})\end{array}$ & $\begin{array}{c}\mathbf{C u} \\
(\mathrm{mg} / \mathrm{kg})\end{array}$ & $\begin{array}{c}\mathbf{M n} \\
(\mathrm{mg} / \mathrm{kg})\end{array}$ & $\begin{array}{c}\mathbf{N i} \\
(\mathrm{mg} / \mathrm{kg})\end{array}$ & $\begin{array}{c}\mathbf{P b} \\
(\mathrm{mg} / \mathrm{kg})\end{array}$ & $\begin{array}{c}\mathbf{Z n} \\
(\mathrm{mg} / \mathrm{kg})\end{array}$ \\
\hline 8,5 & 9,6 & 5,6 & 0,31 & 346 & 0,9 & 0,005 & 3,43 & 12,32 & 1,03 & 1,4 & 19,64 \\
\hline
\end{tabular}

Fuente: Amezcua-Ávila et al. (2020)

\section{Metodología}

Metodología de la fitorremediacion aplicada al suelo del jal de mina

La metodología para el sembrado de la especie vegetal Lolium perenne y Poa pratensis tuvo el siguiente proceso: 
Primero, se realizó la siembra de la especie vegetal Lolium perenne y Poa pratensis, a las dos especies se adicionó composta, la siembra se efectuó en época de lluvia y a una densidad de 357 kg/h (Amezcua-Ávila et al., 2020).

Segundo, la cosecha de Lolium perenne y Poa pratensis se realizó a los 103 días y 80 días, respectivamente, siendo separadas en parte aérea y raíz, tanto las hojas como las raíces fueron secadas en una estufa a $75^{\circ} \mathrm{C}$ para determinar su respectiva masa (AmezcuaÁvila et al., 2020).

Tercero, mediante espectrometría de absorción atómica se cuantifico la concentración de metales pesados en la raíz y hojas de cada especie vegetal (Amezcua-Ávila et al., 2020).

Cuarto, se realizó la evaluación de los datos obtenidos.

\section{Evaluación de los datos obtenidos de las especies Lolium perenne y Poa pratensis}

En la Tabla 4 se presentan las propiedades de concentraciones de $\mathrm{Pb}$ y $\mathrm{Cd}$, factor de bioconcentración (FBC), factor de translocación (FT) y masa total de las especies Lolium perenne y Poa pratensis. A partir de las propiedades se realizó el respectivo análisis.

\section{Tabla 4}

Propiedades de las especies Lolium perenne y Poa pratensis

\begin{tabular}{|c|c|c|c|c|c|c|c|c|c|c|c|c|c|c|}
\hline \multirow{3}{*}{$\begin{array}{l}\text { Parte } \\
\text { de la } \\
\text { planta }\end{array}$} & \multicolumn{7}{|c|}{ Lolium perenne } & \multicolumn{7}{|c|}{ Poa pratensis } \\
\hline & \multicolumn{2}{|c|}{ Concentración } & \multicolumn{2}{|c|}{ FBC } & \multicolumn{2}{|c|}{ FT } & \multirow{2}{*}{$\begin{array}{c}\text { Masa } \\
\text { total (g) }\end{array}$} & \multicolumn{2}{|c|}{ Concentración } & \multicolumn{2}{|c|}{ FBC } & \multicolumn{2}{|c|}{ FT } & \multirow{2}{*}{$\begin{array}{c}\text { Masa } \\
\text { total } \\
(\mathrm{g})\end{array}$} \\
\hline & $\begin{array}{c}\mathrm{Pb} \\
(\mathrm{mg} / \mathrm{kg})\end{array}$ & $\begin{array}{c}\mathrm{Cd} \\
(\mathrm{mg} / \mathrm{kg})\end{array}$ & $\mathrm{Pb}$ & $\mathrm{Cd}$ & $\mathrm{Pb}$ & $\mathrm{Cd}$ & & $\begin{array}{c}\mathrm{Pb} \\
(\mathrm{mg} / \mathrm{kg})\end{array}$ & $\underset{(\mathrm{mg} / \mathrm{kg})}{\mathrm{Cd}}$ & $\mathrm{Pb}$ & $\mathrm{Cd}$ & $\mathrm{Pb}$ & $\mathrm{Cd}$ & \\
\hline Hojas & 28,5 & 6,25 & 2 & 8,56 & \multirow{2}{*}{0,5} & \multirow{2}{*}{1,8} & & 27,1 & 2,1 & 2 & 2,9 & \multirow{2}{*}{0,46} & \multirow{2}{*}{0,76} & \\
\hline Raíces & 57,1 & 3,4 & 4 & 4,66 & & & & 59,15 & 2,75 & 4,12 & 3,77 & & & \\
\hline Total & 85,6 & 9,65 & & & & & 773,96 & 86,25 & 4,85 & & & & & 118,7 \\
\hline
\end{tabular}

Fuente: Elaboración propia basada en Amezcua-Ávila et al. (2020)

La concentración de metales en las dos plantas investigadas muestra que la mayor concentración de $\mathrm{Pb}$ y $\mathrm{Cd}$ se localizan en sus raíces. La planta Lolium perenne tuvo valores de $\mathrm{Pb}$ de $57,1 \mathrm{mg} / \mathrm{kg}$ y $\mathrm{Cd}$ de $3,4 \mathrm{mg} / \mathrm{kg}$, mientas que la especie Poa pratensis tuvo valores de $\mathrm{Pb}$ de $59,15 \mathrm{mg} / \mathrm{kg}$ y $\mathrm{Cd}$ de $2,75 \mathrm{mg} / \mathrm{kg}$. La especie Lolium perenne contiene la mayor concentración total de $\mathrm{Pb}$ y Cd, con valores de $85,6 \mathrm{mg} / \mathrm{kg}$ y 9,65 mg/kg, respectivamente, en el proceso de fitorremediacion.

La especie vegetal Lolium perenne presentó valores totales de concentración de $\mathrm{Pb}$ y $\mathrm{Cd}$ de $85,6 \mathrm{mg} / \mathrm{kg}$ y $9,65 \mathrm{mg} / \mathrm{kg}$, respectivamente, que en comparación con la especie Poa 
pratensis con valores de $\mathrm{Pb}$ de $86,25 \mathrm{mg} / \mathrm{kg}$ y $\mathrm{Cd}$ de 4,85 , tiene mejores valores totales de concentración de $\mathrm{Pb}$ y $\mathrm{Cd}$ en sus estructuras orgánicas.

La planta Lolium perenne presentó un FBC de Pb en raíz de 4 con FT de 0,5; los cuales son mayores a 1 y menores a 1 , respectivamente; lo que da como resultado que la planta utilizó una técnica de fitoestabilización para inmovilizar $\mathrm{Pb}$ en la raíz.

La planta Lolium perenne presentó un FBC de Cd en la parte aérea de 8,56 con FT de 1,8, los cuales son mayores a 1 , lo que da como resultado que la planta utilizó una técnica de fitoextracción para absorber $\mathrm{Cd}$ en sus hojas.

Por la evaluación de los datos, se considera que la especie vegetal Lolium perenne es una planta de fitoestabilización de $\mathrm{Pb}$ y $\mathrm{Cd}$.

La planta Poa pratensis presentó un FBC de Pb en raíz de 2 con FT de 0,46; los cuales son mayores a 1 y menores a 1 , respectivamente; lo que da como resultado que la planta utilizó una técnica de fitoestabilización para inmovilizar $\mathrm{Pb}$ en la raíz.

La planta Poa pratensis presentó un FBC de Cd en raíz de 3,77 con FT de 0,76; los cuales son mayores a 1 y menores a 1 , respectivamente; lo que da como resultado que la planta utilizó una técnica de fitoestabilización para inmovilizar Cd en la raíz.

Como resultado de la investigación realizada por Amezcua-Ávila et al. (2020) se menciona que la especie vegetal Lolium perenne es adecuada para la extracción de metales pesados como el $\mathrm{Pb}$ y $\mathrm{Cd}$ y recomienda su utilización en la revegetación de jales de mina.

En cuento a la producción de masa total de la planta Lolium perenne con 773,96 g, la investigación de Amezcua-Ávila et al. (2020), consideró que se produjo una adecuada cantidad, a pesar de las condiciones desfavorables que se tuvo en el jal de mina Dos Carlos, por lo que los autores recomiendan su utilización con fines de revegetación.

La investigación desarrollada por Amezcua-Ávila et al. (2020), recomienda la adición de composta y la siembra de la especie vegetal Lolium perenne como saneamiento de jales de mina.

Para el posterior análisis de resultados, donde se seleccionará la especie vegetal con la mejor retención de $\mathrm{Pb}$ y $\mathrm{Cd}$ en sus órganos, se escogió la especie Lolium perenne, debido a que posee las mejores propiedades en comparación con la especie Poa pratensis. 
Fitorremediación mediante maíz en suelos degradados por metales pesados en el Valle De Mantaro

La fitorremediación con la especie vegetal Zea mays (maíz) tuvo varias experimentaciones, de las cuales, solo dos son citadas en este ártico, debido a que presentaron los mejores resultados en el proceso de extracción de $\mathrm{Pb}$ y Cd. Esta se llevó a cabo en los suelos agrícolas de la localidad de Muqui y Mantaro, en el Valle de Mantaro, con el propósito de remediar su contaminación por metales pesados. Para mejorar las características de estos suelos y con ello la absorción de metales, fue adicionado enmienda orgánica. La experimentación siguió una metodología, y una vez cumplido el ciclo de crecimiento, se procedió a evaluar la concentración de $\mathrm{Pb}$ y $\mathrm{Cd}$.

\section{Características del suelo agrícola de la localidad de Muqui y Mantaro}

La fitorremediacion en el suelo agrícola de las localidades de Muqui y Mantaro, se pretende llevar a cabo para solubilizar los elementos contaminantes como $\mathrm{Pb}$ y $\mathrm{Cd}$, que se acumularon en el suelo debido a su irrigación con las aguas contaminas del rio Mantaro, producto de los desechos mineros arrojados al mismo (Munive et al., 2018).

El suelo agrícola de la localidad de Muqui y Mantaro, está ubicado en el Valle de Mantaro, Perú, a una altura de 3340 msnm y 3349 m s.n.m., respectivamente (Munive et al., 2018). Las características del suelo agrícola fueron obtenidas mediante un análisis realizado en la Universidad Nacional Agraria La Molina y son presentadas en la Tabla 5.

Tabla 5

Características del suelo agrícola de la localidad de Muqui y Mantaro

\begin{tabular}{lcc}
\hline Características & $\begin{array}{c}\text { Valores del suelo de } \\
\text { Mantaro }\end{array}$ & $\begin{array}{c}\text { Valores del suelo de } \\
\text { Muqui }\end{array}$ \\
\hline Textura & Franco arcilloso & Franco arenoso \\
$\mathrm{pH} 1: 1$ & 7,3 & 7,85 \\
$\mathrm{C} . \mathrm{E}(\mathrm{dS} / \mathrm{m})$ & 0,18 & 0,38 \\
$\mathrm{CaCO} \%$ & 0,00 & 15,7 \\
$\mathrm{M} . \mathrm{O} \%$ & 3,56 & 2,3 \\
$\mathrm{P}$ disponible $(\mathrm{mg} / \mathrm{kg})$ & 29,7 & 15,8 \\
$\mathrm{~K}$ disponible $(\mathrm{mg} / \mathrm{kg})$ & 239 & 149 \\
$\mathrm{CIC}$ total $(\mathrm{meq} / \mathrm{100})$ & 20 & 11,2 \\
$\mathrm{Saturación} \mathrm{de} \mathrm{bases} \mathrm{\%}$ & 100 & 100 \\
$\mathrm{Ca}(\mathrm{mg} / \mathrm{kg})$ & 17,08 & 9,57 \\
$\mathrm{Mg}(\mathrm{mg} / \mathrm{kg})$ & 2,37 & 1,23 \\
$\mathrm{~K}(\mathrm{mg} / \mathrm{kg})$ & 0,47 & 0,33 \\
$\mathrm{Na}(\mathrm{mg} / \mathrm{kg})$ & 0.09 & 0,07 \\
$\mathrm{~Pb}(\mathrm{mg} / \mathrm{kg})$ & 208,24 & 1174,44 \\
$\mathrm{Cd}(\mathrm{mg} / \mathrm{kg})$ & 6,76 & 8,26 \\
\hline
\end{tabular}

Fuente: Munive et al. (2018) 
Enmienda orgánica agregada al suelo agrícola de la localidad de Muqui y Mantaro

La adición de enmienda orgánica para mejorar el crecimiento de la especie vegetal Zea mays (maíz) se la realizó a partir del producto vermicompost (Munive et al., 2018). En la tabla 6 se presentan las características de la enmienda orgánica vermicompost agregada tanto al suelo agrícola de la localidad de Muqui como a la de Mantaro.

\section{Tabla 6}

Características de la enmienda orgánica vermicompost agregada al suelo de Muqui y Mantaro

\begin{tabular}{lc} 
Características & Vermicompost \\
\hline $\mathrm{pH}$ & 6,82 \\
$\mathrm{C} . \mathrm{E}(\mathrm{dS} / \mathrm{m})$ & 1,87 \\
$\mathrm{M} . \mathrm{O} \%$ & 29,55 \\
$\mathrm{~N} \%$ & 1,1 \\
$\mathrm{P}_{2} \mathrm{O}_{5} \%$ & 1,09 \\
$\mathrm{~K}_{2} \mathrm{O} \%$ & 0,38 \\
$\mathrm{CaO} \%$ & 2,3 \\
$\mathrm{MgO} \%$ & 0,49 \\
$\mathrm{Hd} \%$ & 39,74 \\
$\mathrm{Na} \%$ & 0,02 \\
$\mathrm{~Pb}(\mathrm{mg} / \mathrm{kg})$ & 35,1 \\
$\mathrm{Cd}(\mathrm{mg} / \mathrm{kg})$ & 39,25 \\
\hline
\end{tabular}

Fuente: Munive et al. (2018)

Metodología de la fitorremediación aplicada al suelo de la localidad de Muqui y Mantaro

La investigación se llevó a cabo en la Universidad Nacional Agraria La Molina, ubicada a 230 m s.n.m., en la provincia de Lima, con una temperatura promedio de 14,6 a 28,7 ${ }^{\circ} \mathrm{C}$. A este centro de investigación fue trasladado cierta cantidad de suelo de la localidad de Muqui y de Mantaro, en donde se realizó el sembrado de maíz a través de la siguiente metodología:

Primero, se realizó la siembra de la especie vegetal Zea mays con la adición de enmienda orgánica vermicompost en tres macetas, con capacidad de $4 \mathrm{~kg}$ cada una. Al inicio en cada maceta fueron colocadas cinco semillas de maíz que posteriormente a su germinación y crecimiento, fueron dejadas solo tres plantas por maceta (Munive et al., 2018).

Segundo, una vez que la planta alcanzó la madurez fisiológica se realizó la cosecha, obteniéndose por separado hojas, tallos y raíces, para posteriormente obtener su masa, el secado se lo realizó en una estufa, en un tiempo de 24 horas y a una temperatura de $75^{\circ} \mathrm{C}$ (Munive et al., 2018). 
Tercero, con las hojas, tallos y raíces de la especie vegetal, se procedió a la cuantificación de la concentración de metales pesados (Munive et al., 2018).

Cuarto, se realizó la evaluación de los datos obtenidos.

\section{Evaluación de los datos obtenidos}

En la tabla 7 se presentan las propiedades de concentraciones de $\mathrm{Pb}$ y $\mathrm{Cd}$, factor de bioconcentración (FBC), factor de translocación (FT) y masa total de la planta de maíz, tanto para el suelo agrícola de Muqui como para el de Mantaro. A partir de las propiedades obtenidas se realizó el respectivo análisis.

\section{Tabla 7}

Propiedades de la planta de maíz sembrada en el suelo agrícola de Muqui y Mantaro

\begin{tabular}{|c|c|c|c|c|c|c|c|c|c|c|c|c|c|c|}
\hline \multirow{3}{*}{$\begin{array}{l}\text { Parte } \\
\text { de la } \\
\text { planta }\end{array}$} & \multicolumn{7}{|c|}{ Planta de maíz sembrada en el suelo de Muqui } & \multicolumn{7}{|c|}{ Planta de maíz sembrada en el suelo de Mantaro } \\
\hline & \multicolumn{2}{|c|}{ Concentración } & \multicolumn{2}{|c|}{ FBC } & \multicolumn{2}{|c|}{ FT } & \multirow{2}{*}{$\begin{array}{l}\text { Masa } \\
\text { total }(\mathrm{g})\end{array}$} & \multicolumn{2}{|c|}{ Concentración } & \multicolumn{2}{|c|}{ FBC } & \multicolumn{2}{|c|}{ FT } & \multirow{2}{*}{$\begin{array}{c}\text { Masa } \\
\text { total } \\
(\mathrm{g})\end{array}$} \\
\hline & $\underset{(\mathrm{mg} / \mathrm{kg})}{\mathrm{Pb}}$ & $\underset{(\mathrm{mg} / \mathrm{kg})}{\mathrm{Cd}}$ & $\mathrm{Pb}$ & $\mathrm{Cd}$ & $\mathrm{Pb}$ & $\mathrm{Cd}$ & & $\begin{array}{c}\mathrm{Pb} \\
(\mathrm{mg} / \mathrm{kg})\end{array}$ & $\underset{(\mathrm{mg} / \mathrm{kg})}{\mathrm{Cd}}$ & $\mathrm{Pb}$ & $\mathrm{Cd}$ & $\mathrm{Pb}$ & $\mathrm{Cd}$ & \\
\hline Hojas & 10,3 & 0,25 & \multirow[b]{2}{*}{0,01} & \multirow[b]{2}{*}{0,05} & & & & 9,25 & 0,45 & \multirow[b]{2}{*}{0,07} & \multirow[b]{2}{*}{0,11} & & \multirow{3}{*}{0,06} & \\
\hline Tallos & 6,03 & 0,23 & & & 0,04 & 0,07 & & 4,38 & 0,38 & & & 0,24 & & \\
\hline Raíces & 379,5 & 7,23 & 0,22 & 0,8 & & & & 56,04 & 13,31 & 0,3 & 1,84 & & & \\
\hline Total & 395,83 & 7,71 & & & & & 64,58 & 69,67 & 14,14 & & & & & 99,55 \\
\hline
\end{tabular}

Fuente: Elaboración propia basada en Munive et al. (2018)

La concentración de metales en la planta de maíz en los suelos de la localidad de Muqui y Mantaro, muestra que la mayor concentración de $\mathrm{Pb}$ y Cd se localizan en sus raíces. La planta de maíz sembrada en el suelo de Muqui tuvo valores de $\mathrm{Pb}$ de $379,5 \mathrm{mg} / \mathrm{kg}$ y Cd de 7,23 mg/kg, mientras que cuando se la sembró en el suelo de Mantaro tuvo valores de $\mathrm{Pb}$ de $56,04 \mathrm{mg} / \mathrm{kg}$ y Cd de $13,31 \mathrm{mg} / \mathrm{kg}$. Por lo tanto, la especie sembrada en el suelo de Muqui realizó la mayor absorción de $\mathrm{Pb}$ y $\mathrm{Cd}$ en el proceso de fitorremediacion.

La planta de maíz sembrada en la localidad de Muqui tiene la mayor concentración total de $\mathrm{Pb}(395,83 \mathrm{mg} / \mathrm{kg})$ respecto al valor de la planta sembrada en el suelo de Mantaro $(69,67 \mathrm{mg} / \mathrm{kg})$. En cambio, la mayor concentración de Cd la tuvo la planta del suelo de Mantaro con un valor de 14,14 mg/kg.

La planta de maíz sembrada en el suelo de Muqui presentó un FBC de Pb en la parte aérea de 0,01 con FT de 0,04, los cuales son menores a 1, lo que da como resultado que la planta utilizó una técnica de fitoestabilización para inmovilizar $\mathrm{Pb}$ en la raíz. 
La planta de maíz sembrada en el suelo de Muqui presentó un FBC de Cd en la parte aérea de 0,05 con FT de 0,07, los cuales son menores a 1, lo que da como resultado que la planta utilizó una técnica de fitoestabilización para inmovilizar Cd en la raíz.

Por la evaluación de los datos, se considera que la planta de maíz, sembrada en el suelo de la localidad de Muqui, es una planta de fitoestabilización de $\mathrm{Pb}$ y Cd.

La planta de maíz sembrada en el suelo de Mantaro presentó un FBC de $\mathrm{Pb}$ en la parte aérea de 0,07 con FT de 0,24, los cuales son menores a 1, lo que da como resultado que la planta utilizó una técnica de fitoestabilización para inmovilizar $\mathrm{Pb}$ en la raíz.

La planta de maíz sembrada en el suelo de Mantaro presentó un FBC de Cd en raíz de 1,84 con FT de 0,06; siendo mayor a 1 y menor a 1, respectivamente; lo que da como resultado que la planta utilizó una técnica de fitoestabilización para inmovilizar $\mathrm{Cd}$ en la raíz.

La planta sembrada en el suelo de la localidad de Muqui presentó un valor de desarrollo de masa de 64,58 g con una concentración de $\mathrm{Pb}$ de 395,83 mg/kg, mientras que la sembrada en el suelo de Mantaro presentó un valor de desarrollo de masa de 99,55 g con una concentración de $\mathrm{Pb}$ de 69,67 mg/kg, esto demuestra que a mayor cantidad de $\mathrm{Pb}$ absorbido por la planta de maíz menor va a ser su desarrollo.

La investigación desarrollada para la fitorremediacion con maíz en el suelo degradado por metales pesados $\mathrm{Pb}$ y $\mathrm{Cd}$, determinó que la planta de maíz es la adecuada para la absorción de metales por la técnica de fitoestabilización (Munive et al., 2018).

La adición de la enmienda orgánica vermicompost contribuyen a la solubilizarían de los metales pesados plomo y cadmio en el suelo agrícola, con lo cual se facilita una mejor absorción de metales a través de la raíz de la planta de maíz (Munive et al., 2018).

Una vez obtenidos resultados favorables de extracción de $\mathrm{Pb}$ y $\mathrm{Cd}$, en el suelo agrícola de la localidad de Muqui, mediante la planta de maíz, se procederá a la culminación del ciclo vegetativo con la siembra de la especie vegetal a nivel de campo (Munive et al., 2018).

Para el posterior análisis de resultados, donde se seleccionará la especie vegetal con la mejor retención de $\mathrm{Pb}$ y $\mathrm{Cd}$ en sus órganos, se escogió la planta de maíz sembrada en la localidad de Muqui, debido a que posee las mejores propiedades en comparación con sembrada en la localidad de Mantaro. 
Fitorremediación mediante Pennisetum Setaceum y Tradescantia Pallida en el suelo del Morro de Moravia

La fitorremediación con las especies vegetales Pennisetum setaceum y Tradescantia pallida, en el suelo del Morro de Moravia, tuvo varias experimentaciones, tanto en diferentes parcelas como en tiempos de cosecha. Para este artículo se seleccionó la experimentación en la parcela B con un tiempo de cosecha de 12 meses, debido a que su concentración de $\mathrm{Pb}$ y Cd fueron elevadas, en comparación con las parcelas A y C. La fitorremediacion pretende reducir los residuos de metales en el suelo, evidenciándose en sus características. Para la siembra y cosecha se siguió una metodología que permitió evaluar la concentración de $\mathrm{Pb}$ y $\mathrm{Cd}$.

\section{Características del suelo del Morro de Moravia}

La fitorremediación con Pennisetum setaceum y Tradescantia pallida en el suelo de la localidad del Morro de Moravia, Colombia, fue realizado para evaluar el potencial fitorremediador de las dos especies vegetales. Siendo sembradas en la localidad como parte de una solución paisajística y ambiental, debido a que la localidad yacía un antiguo botadero de basura a cielo abierto (Pajoy, 2017).

La muestra para la determinación de las características del suelo de la parcela B de la localidad del Morro de Moravia fue tomada aproximadamente a una profundidad de 20 cm y a una altura de 1460 m s.n.m. (Pajoy, 2017). Las características del suelo de la parcela B son presentadas en la tabla 8 .

\section{Tabla 8}

Características del suelo de la parcela $B$

\begin{tabular}{lc}
\hline Características & Valores \\
\hline Arcilla \% & 68 \\
Limo \% & 22 \\
Arena \% & 10 \\
pH & 8 \\
$\mathbf{C I C E}$ & 30,7 \\
$\mathbf{P}$ & 125 \\
$\mathbf{N}-N_{3} \%$ & 21 \\
$\mathbf{N}-\mathbf{N H}_{\mathbf{4}} \%$ & 14 \\
$\mathbf{M O} \%$ & 8,7 \\
$\mathbf{C d}(\mathrm{mg} / \mathrm{g})$ & 0,77 \\
$\mathbf{C r}(\mathrm{mg} / \mathrm{g})$ & 335,35 \\
$\mathbf{N i}(\mathrm{mg} / \mathrm{g})$ & 474,87 \\
$\mathbf{P b}(\mathrm{mg} / \mathrm{g})$ & 236,39 \\
\hline \multicolumn{2}{c}{ Fuente: Pajoy $(2017)$}
\end{tabular}


Metodología de fitorremediación aplicada al suelo de la localidad del Morro de Moravia La siembra, cosecha y recolección de las muestras se lo realizó en la localidad del Morro de Moravia, mientras que los exámenes para la cuantificación de datos fueron realizaron en la Universidad Nacional de Colombia y en la Universidad de Antioquia. La metodología para la siembra y cosecha del proceso de fitorremediacion en la parcela B fue realizada de la siguiente forma:

Primero, al ser un suelo antropogénico, debido a que fue un antiguo botadero de basura a cielo abierto, fueron removidos los residuos que estaban en la parcela $\mathrm{B}$, una vez limpio se procedió a sembrar 33 especies vegetales de Pennisetum setaceum y 33 especies de Tradescantia pallida (Pajoy, 2017).

Segundo, se realizó la cosecha de dos especies de Pennisetum setaceum y dos especies de Tradescantia pallida, en un tiempo de 12 meses, para posteriormente separar la raíz de la parte aérea (Pajoy, 2017).

Tercero, se procedió a determinar, en el Laboratorio del Grupo Interdisciplinario de Estudios Moleculares GIEM de la Universidad de Antioquia, la concentración de metales pesados en las raíces y parte aérea de las especies vegetales (Pajoy, 2017).

Cuarto, se realizó la evaluación de los datos obtenidos.

\section{Evaluación de los datos obtenidos}

En la tabla 9 se presentan las propiedades de concentraciones de $\mathrm{Pb}$ y $\mathrm{Cd}$, factor de bioconcentración (FBC) y factor de translocación (FT) de las especies vegetales Pennisetum setaceum y Tradescantia pallida sembradas en la parcela B. A partir de las propiedades obtenidas se realizó el respectivo análisis.

\section{Tabla 9}

Propiedades de las especies vegetales Pennisetum setaceum y Tradescantia pallida

\begin{tabular}{|c|c|c|c|c|c|c|c|c|c|c|c|c|c|c|}
\hline \multirow{3}{*}{$\begin{array}{c}\text { Parte } \\
\text { de la } \\
\text { planta }\end{array}$} & \multicolumn{7}{|c|}{ Pennisetum setaceum } & \multicolumn{7}{|c|}{ Tradescantia pallida } \\
\hline & \multicolumn{2}{|c|}{ Concentración } & \multicolumn{2}{|c|}{$\mathrm{FBC}$} & \multicolumn{2}{|c|}{ FT } & \multirow{2}{*}{$\begin{array}{l}\text { Masa } \\
\text { total }(\mathrm{g})\end{array}$} & \multicolumn{2}{|c|}{ Concentración } & \multicolumn{2}{|c|}{ FBC } & \multicolumn{2}{|c|}{ FT } & \multirow{2}{*}{$\begin{array}{c}\text { Masa } \\
\text { total } \\
(\mathrm{g})\end{array}$} \\
\hline & $\begin{array}{c}\mathrm{Pb} \\
(\mathrm{mg} / \mathrm{kg})\end{array}$ & $\begin{array}{c}\mathrm{Cd} \\
(\mathrm{mg} / \mathrm{kg})\end{array}$ & $\mathrm{Pb}$ & $\mathrm{Cd}$ & $\mathrm{Pb}$ & $\mathrm{Cd}$ & & $\begin{array}{c}\mathrm{Pb} \\
(\mathrm{mg} / \mathrm{kg})\end{array}$ & $\begin{array}{c}\mathrm{Cd} \\
(\mathrm{mg} / \mathrm{kg})\end{array}$ & $\mathrm{Pb}$ & $\mathrm{Cd}$ & $\mathrm{Pb}$ & $\mathrm{Cd}$ & \\
\hline Hojas & 9,8 & 0,003 & 0,04 & 0,004 & \multirow{2}{*}{0,1} & \multirow{2}{*}{0} & & 114,1 & 4,2 & 0,5 & 5,5 & \multirow{2}{*}{0,53} & \multirow{2}{*}{0,2} & \\
\hline Raíces & 164,6 & 9,7 & 0,7 & 12,6 & & & & 216,8 & 17,03 & 0,92 & 22,1 & & & \\
\hline Total & 174,4 & 9,703 & & & & & ---- & 330,9 & 21,23 & & & & & ---- \\
\hline
\end{tabular}

Fuente: Elaboración propia basada en Pajoy (2017) 
La concentración de metales en las dos plantas investigadas muestra que la mayor concentración de $\mathrm{Pb}$ y $\mathrm{Cd}$ se localizan en sus raíces. La planta Tradescantia pallida tuvo valores de $\mathrm{Pb}$ de $216,8 \mathrm{mg} / \mathrm{kg}$ y Cd de $17,03 \mathrm{mg} / \mathrm{kg}$, mientas que la especie Pennisetum setaceum tuvo valores de $\mathrm{Pb}$ de $164,6 \mathrm{mg} / \mathrm{kg}$ y $\mathrm{Cd}$ de $9,7 \mathrm{mg} / \mathrm{kg}$, siendo la especie Tradescantia pallida la que mayor absorción de $\mathrm{Pb}$ y $\mathrm{Cd}$ realizó en el proceso de fitorremediacion.

La especie vegetal Tradescantia pallida presentó valores totales de concentración de $\mathrm{Pb}$ y Cd de 330,9 mg/kg y 21,23 mg/kg, respectivamente, que en comparación con la especie Pennisetum setaceum con valores de $\mathrm{Pb}$ de $174,4 \mathrm{mg} / \mathrm{kg}$ y $\mathrm{Cd}$ de 9,703 , se tiene que la especie vegetal concentra mayores valores de $\mathrm{Pb}$ y $\mathrm{Cd}$ en sus estructuras orgánicas.

La planta Tradescantia pallida presentó un FBC de Pb en la parte aérea de 0,5 con FT de 0,53 , los cuales son menores a 1 , lo que da como resultado que la planta utilizó una técnica de fitoestabilización para inmovilizar Pb en la raíz.

La planta Tradescantia pallida presentó un FBC de Cd en la raíz de 22,1 con FT de 0,2; los cuales son mayores a 1 y menores a 1 , respectivamente; lo que da como resultado que la planta utilizó una técnica de fitoestabilización para inmovilizar Cd en la raíz.

Por la evaluación de los datos, se considera que de la especie vegetal Tradescantia pallida, sembrada en la parcela B de la localidad del Morro de Moravia, es una planta de fitoestabilización de $\mathrm{Pb}$ y $\mathrm{Cd}$.

La planta Pennisetum setaceum presentó un $\mathrm{FBC}$ de $\mathrm{Pb}$ en la parte aerea de 0,04 con FT de 0,1 , los cuales son menores a 1 , lo que da como resultado que la planta utilizó una técnica de fitoestabilización para inmovilizar $\mathrm{Pb}$ en la raíz.

La planta Pennisetum setaceum presentó un FBC de Cd en la raíz de 12,6 con FT de 0; los cuales son mayores a 1 y menores a 1 , respectivamente; lo que da como resultado que la planta utilizó una técnica de fitoestabilización para inmovilizar Cd en la raíz.

Como resultado de la investigación realizada por Pajoy (2017), se menciona que la especie vegetal Tradescantia pallida posee potencial fitorremediador en sustratos con presencia de concentraciones de cadmio y plomo.

Para el posterior análisis de resultados, donde se seleccionará la especie vegetal con la mejor retención de $\mathrm{Pb}$ y $\mathrm{Cd}$ en sus órganos, se escogió la especie Tradescantia pallida, debido a que posee las mejores propiedades en comparación con la especie Pennisetum setaceum. 


\section{Resultados}

En la tabla 10 se presentan las propiedades de concentraciones, factor de bioconcentración de la raíz (FBCraíz) y factor de translocación (FT) de $\mathrm{Pb}$ y $\mathrm{Cd}$, además del tiempo de cosecha, de las especies vegetales Lolium perenne, Maíz y Tradescantia pallida, para su respectivo análisis.

\section{Tabla 10}

Propiedades de las especies vegetales Lolium perenne, Maíz y Tradescantia pallida

\begin{tabular}{lcccccc}
\hline & $\begin{array}{c}\text { Lolium } \\
\text { perenne }\end{array}$ & Maíz & $\begin{array}{c}\text { Tradescantia } \\
\text { pallida }\end{array}$ & $\begin{array}{c}\text { Lolium } \\
\text { perenne }\end{array}$ & $\begin{array}{c}\text { Maíz } \\
\text { Propiedades }\end{array}$ & $\begin{array}{c}\text { Tradescantia } \\
\text { pallida }\end{array}$ \\
\hline & $\mathrm{Pb}$ & $\mathrm{Pb}$ & $\mathrm{Pb}$ & $\mathrm{Cd}$ & $\mathrm{Cd}$ & $\mathrm{Cd}$ \\
\hline FBC $_{\text {ráí }}$ & 4 & 0,22 & 0,92 & 4,66 & 0,8 & 22,1 \\
FT & 0,5 & 0,04 & 0,53 & 1,8 & 0,07 & 0,2 \\
Concentración en parte aérea (mg/kg) & 28,5 & 16,33 & 114,1 & 6,25 & 0,48 & 4,2 \\
Concentración en raíces (mg/kg) & 57,1 & 379,5 & 216,8 & 3,4 & 7,23 & 17,03 \\
Concentración total (mg/kg) & 85,6 & 395,83 & 330,9 & 9,65 & 7,71 & 21,23 \\
Tiempo de cosecha (días) & 103 & 134 & 365 & 103 & 134 & 365 \\
\hline
\end{tabular}

Fuente: Elaboración propia basada en Amezcua-Ávila et al. (2020), Munive et al. (2018) y Pajoy (2017)

Como resultado de la aplicación de las técnicas de fitorremediacion en las tres especies vegetales, se obtiene que la especie Tradescantia pallida al tener valores de concentración total de $\mathrm{Pb}$ de $330,9 \mathrm{mg} / \mathrm{kg}$ y Cd $21,23 \mathrm{mg} / \mathrm{kg}, \mathrm{FBC}_{\text {raíz }}$ de 0,92 y $\mathrm{FT}$ de 0,53 para $\mathrm{Pb}$, $\mathrm{FBC}_{\text {raíz }}$ de 22,1 y FT de 0,2 para Cd, alcanzó las mejores propiedades para retener $\mathrm{Pb}$ y Cd por la técnica de fitoestabilización, en comparación con las plantas de Maíz y la Tradescantia pallida.

La planta Lolium perenne presentó un valor de $\mathrm{FBC}_{\text {raíz }}$ de $\mathrm{Cd}$ de 4,66 con FT de 1,8, dando como resultado que la planta sembrada tiene potencial para realizar procesos de fitoextracción de Cd.

La mayor concentración de $\mathrm{Pb}$ se acumula en las raíces de las especies vegetales, teniendo valores para Lolium perenne de $57,1 \mathrm{mg} / \mathrm{kg}$, Maíz de $379,5 \mathrm{mg} / \mathrm{kg}$,

Tradescantia pallida de $216,8 \mathrm{mg} / \mathrm{kg}$, lo cual es característica de plantas fitoestabilizadoras.

\section{Conclusiones}

- La especie vegetal Tradescantia pallida a pesar de que fue sembrada en un suelo antropogénico con elevado contenido de contaminación, ya que fue un antiguo botadero de basura a cielo abierto, logró acumular valores de concentración de $\mathrm{Pb}$ 
en la parte aérea de $114,1 \mathrm{mg} / \mathrm{kg}$ y de Cd en la raíz de $17,03 \mathrm{mg} / \mathrm{kg}$, que en comparación con las otras especies vegetales investigadas, tienen una mejor concentración de metales, razón por la cual, se la puede seleccionar para su desarrollo en cualquier suelo contaminado con $\mathrm{Pb}$ y $\mathrm{Cd}$.

- La selección de la especie vegetal Tradescantia pallida en procesos de fitorremediacion de suelos contaminados con $\mathrm{Pb}$ y $\mathrm{Cd}$, presenta un ahorro económico de insumos agrícolas, ya que la misma no requirió la adición de abono o enmienda orgánica para su crecimiento.

- La planta de maíz con valores de concentración total de $\mathrm{Pb}$ de 395,83 mg/kg y Cd de $7,71 \mathrm{mg} / \mathrm{kg}$, podría ser una alternativa para remediación de suelos agrícolas degradados, ya que presentó un menor tiempo de cosecha, 134 días, lo cual influye en la disponibilidad del suelo después de ser descontaminado.

- La adición de abono o enmienda orgánicos contribuye al descenso del $\mathrm{pH}$, lo que incrementó la solubilidad de los metales en la especie vegetal Lolium perenne y Maíz.

\section{Referencias Bibliográficas}

Ashraf, M., Ozturk, M., \& Ahmad, M. S. A. (Eds.). (2010). Plant adaptation and phytoremediation. New York, NY, USA: Springer.

Ávila González, D. A. (2017). Fitoextracción de suelos contaminados por elementos potencialmente tóxicos en la región de Atlixco, Puebla (Tesis de Maestría). Benemérita Universidad Autónoma de Puebla. México

Amezcua-Ávila, A. V., Hernández-Acosta, E., \& Díaz, V. P. (2020). Fitorremediación de residuos de minas contaminados con metales pesados. Revista Iberoamericana de Ciencias, 7, 79-81.

Jaramillo Navarro, P. A. (2021). Revisión de técnicas de fitorremediación de suelos contaminados con metales pesados provenientes de la actividad minera $e$ industrial (Tesis de Maestría). EPN. Quito.

Lokeshwari, H., \& Chandrappa, G. T. (2006). Impact of heavy metal contamination of Bellandur Lake on soil and cultivated vegetation. Current science, 622-627.

Martínez González, H. (2020). Fitoextracción de elementos tóxicos de suelos de Zacatecas, contaminados con residuos mineros (Tesis de Maestría). Universidad Autónoma Metropolitana. México.

Medina Marcos, K. D., \& Montano Chávez, Y. N. (2014). Determinación del factor de Bioconcentración y Traslocación de metales pesados en el Juncus arcticus Willd. 
Y Cortaderia rudiuscula Stapf, de áreas contaminadas con el pasivo ambiental minero Alianza-Ancash 2013 (Tesis de Ingeniería). Universidad Nacional Santiago Antúnez de Mayolo. Perú.

MineralsUK.

(2022).

World

mineral

statistics

data.

https://www2.bgs.ac.uk/mineralsuk/statistics/wms.cfc?method=searchWMS

Munive Cerrón, R., Loli Figueroa, O., Azabache Leyton, A., \& Gamarra Sánchez, G. (2018). Fitorremediación con Maíz (Zea mays L.) y compost de Stevia en suelos degradados por contaminación con metales pesados. Scientia Agropecuaria, 9(4), 551-560.

Pajoy Muñoz, H. M. (2017). Potencial fitorremediador de dos especies ornamentales como alternativa de tratamiento de suelos contaminados con metales pesados (Tesis de Maestría). Universidad Nacional de Colombia. Medellín.

Rodríguez-Eugenio, N., McLaughlin, M. y Pennock, D. (2019). La contaminación del suelo: una realidad oculta. Roma, FAO.

Volke, T., \& Velasco, J. (2002). Tecnologías de remediación para suelos contaminados. México: INE-SEMARNAT.

Wang, Z., Liu, X., \& Qin, H. (2019). Bioconcentration and translocation of heavy metals in the soil-plants system in Machangqing copper mine, Yunnan Province, China. Journal of Geochemical Exploration, 200, 159-166.

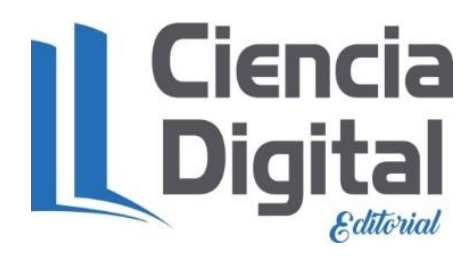




\section{Conciencia}

El artículo que se publica es de exclusiva responsabilidad de los autores y no necesariamente reflejan el pensamiento de la Revista Conciencia Digital.

\section{\Ciencia}

El artículo queda en propiedad de la revista y, por tanto, su publicación parcial y/o total en otro medio tiene que ser autorizado por el director de la Revista Conciencia Digital.
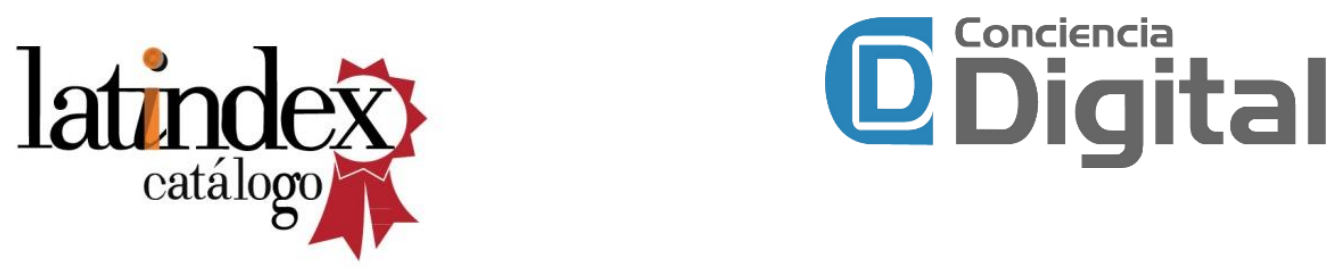

Indexaciones

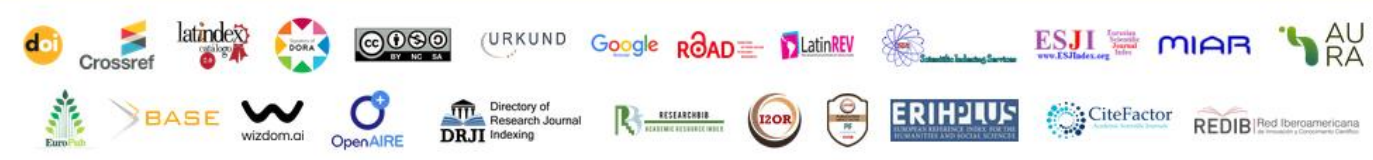

